\title{
An Approach to Planning Services for Bulimia Nervosa
}

\author{
JANET L. TREASURE, NICHOLAS A. TROOP and ANNE WARD
}

How should health care strategists respond to the emergence and subsequent increase in bulimia nervosa over the last two decades? A recent publication on eating disorders from the Office of Health Economics failed to address the costs of bulimia nervosa (West, 1994), citing the nonavailability of figures for counselling or out-patient psychological treatment. However, purchasers of health care need to make provision for bulimia nervosa and a major part of their remit will be an economic evaluation of available treatments. The National Health Service (NHS) itself has no figures on the impact of bulimia nervosa because it has not been coded as a separate illness until recently. Nor are there any current health economic evaluations of treatment for bulimia nervosa. However, it is possible to obtain estimates of costs based on health service research. As money for research is finite, time-limited treatments have typically been the focus of interest. Results from this type of research bear on cost and outcome and, therefore, economic assessment. While these may not provide sufficient data for a rigorous analysis, they have a valuable role in aiding decision making in the NHS, and in suggesting avenues for further health economic evaluation.

There are a number of variables affecting both cost and outcome of treatment. In this paper, we discuss issues relating to type of treatment, dose and level of specialisation of therapist. Our aim is to review available evidence, pointing to the economic implications, and to highlight the need for more formal, planned, economic assessment.

\section{Type and effect of treatment}

Several treatment studies have been done in bulimia nervosa. A meta-analysis of results up to 1985 found an overall effect size of 0.95 (Laessle et al, 1987). However, few of these studies were randomised, controlled trials. Psychological treatment incorporating dietary management was the most effective mode of treatment, and drug treatment had the least effect. In terms of prognostic factors, older patients and those with a longer duration of illness had the poorest outcome. A later metaanalysis of group treatments for bulimia nervosa was undertaken by Fettes \& Peters (1992). They included studies to 1989 but, again, few were randomised. The overall effect size for group treatment was 0.7 . Treatments that involved more hours of therapy per week produced better shortterm outcome.

One problem in comparing treatment studies for bulimia nervosa is that different outcome criteria have often been used. Many researchers use symptom frequency but, as this is not normally distributed, percentage change can be misleading. The more stringent criterion of abstinence from all symptoms (binge eating and purging) is a useful outcome measure. Comparison of abstinence rates with different treatments allows a cost-effectiveness analysis when treatment costs are quoted (see Robinson, 1993a). Abstinence rates ranging from 30 to $60 \%$ have been obtained over the last decade (for a review, see Agras, 1993). Where psychological treatment was used, the therapeutic input has ranged from 15 to 66 sessions. Cognitive-behavioural treatment (CBT) and interpersonal therapy (IPT), in particular, are especially effective (CBT is considered to be the 'gold standard'), while behaviour therapy is not as effective over the long term (Fairburn et al, 1993).

It was hoped that newer antidepressants, such as fluoxetine, would have a greater and more sustained effect than the older tricyclics, but this expectation has not been realised. The 16-week outcome figures for the use of fluoxetine show abstinence from vomiting in $19 \%$ of patients and abstinence from binge eating in $18.3 \%$ (Goldstein et al, 1995). (Overall abstinence rates are not deducible from the data supplied.) The value of maintenance therapy in responders has not been tested.

\section{Dose of treatment}

Treatments that involve a high therapeutic input tend to be more successful (e.g. Fettes \& Peters, 
1992). However, such intensive intervention may not be necessary for everyone and would be wasteful for individuals who do not require it. As they are also costly, treatments aimed at reducing the number of therapist-hours have been developed.

A group psychoeducation intervention of five 90 min lectures (total of $7.5 \mathrm{~h}$ ), with an emphasis on symptom management, produced an abstinence from binge eating and vomiting in $20 \%$ of patients. However, at the 3-month follow-up, psychoeducation was found to be less effective overall than 19 1-h sessions of CBT. Of interest, and worth further investigation, was the observation that the two interventions were equivalent in a less severely affected subgroup (Olmstead et al, 1991). A similar rate of recovery was found in an open evaluation of a guided self-help group (Rathner et al, 1993). Although these interventions still require specialist knowledge, they make the point that a number of patients can recover with considerably less than the standard CBT input of $161-\mathrm{h}$ sessions. Seven and a half hours of group therapy for such patients is considerably more economical than $16 \mathrm{~h}$ of individual therapy per patient.

Our own group has investigated the use of a selftreatment manual for bulimia nervosa in a randomised, controlled trial, which was conducted in two stages (Treasure et al, 1996). In the first stage, the outcome of those given the manual was compared with that of those remaining on the waiting list. No significant reductions in any bulimic symptoms were found in the waiting list group. The manual produced full remission in $22 \%$ of cases, which is an effect size of 0.30 . In the second stage of the study, up to eight sessions of CBT were offered to the group who had failed to make a substantial improvement with the manual alone (manual + CBT was called 'sequential treatment'). These patients were compared to those who had been given a standard 16-session CBT. The mean number of sessions in the sequential group as a whole was 2.8 . In the subgroup who actually received CBT, the mean number of sessions was still only 6.1 , which is a considerable reduction from the standard 16 sessions. There was no significant difference in outcome between the standard CBT and sequential groups, i.e. $30 \%$ abstinence from all bulimic behaviours (binge eating and purging) in both groups (Treasure et al, 1996). The effect size of sequential treatment was 0.48 (at the end of treatment), which compares favourably with effect sizes seen in a comparison of more intensive treatments, where behaviour therapy had an effect size of 0.25, CBT had an effect size of 0.61 and IPT had an effect size of 0.78 (Fairburn et al, 1993). The results of sequential treatment were maintained at 18 months (40\% abstinence in both groups; Treasure et al, 1996), suggesting that the 'dose' of treatment required to produce the same rate of symptom relief can be reduced by more effective methods of delivering information and skills training than are generally employed.

We found that one of the major determinants of outcome with a self-care book was compliance. For example, we found that only $57 \%$ of our patients read more than half the book, $43 \%$ completed two or more exercises and only $26 \%$ sought the assistance of a friend, relative or partner as a "cotherapist/supporter', even though this was explicitly advised (Troop et al, 1996). Forty per cent of those who complied with treatment achieved full remission after 8 weeks with a manual compared with only $5 \%$ of those who did not comply. These figures also highlight the role of ambivalence in determining outcome, and the importance of a motivational aspect to any treatment programme.

However, $50 \%$ of patients given minimal or moderate intensity treatment remain symptomatic at the end of treatment and little improvement occurs even six years after treatment (Fairburn et al, 1995). What additional input is necessary to improve the outcome? One open study partially addressed this question. Psychodynamic psychotherapy was added to CBT, which led to a significant improvement in a group with personality disorders (Johnson et al, 1989). There appeared to be a dosage effect in that patients who improved had an average of 100 sessions during the first year, whereas patients who received an average of 50 sessions remained symptomatic. This is similar to findings in other areas of psychotherapeutic research in which a linear relationship has been found between the logarithm of the number of sessions and the normalised probability of patient improvement (Howard et al, 1993). The argument of diminishing returns for increasing cost may then be invoked, but it is often our impression that patients needing longer term treatments are in fact heavy users of health care and social services. Further studies that address dosage effects along with cost and try to match patient with therapy are clearly indicated.

\section{Level of training for therapists}

A parallel question to the one of treatment dose is: "What is the minimum effective training needed for the management of eating disorders?" Most research has taken place in specialised centres with trained therapists. This may limit the usefulness of findings that one particular treatment is better than 
another, as training places for these 'gold standard' treatments like CBT are limited and require a large investment of resources. It is interesting, therefore, that a social worker without specialist training, using a cognitive-behavioural-based manual, achieved similar outcomes to therapists with CBT training (Cooper et al, 1994). Further investigation of treatments that require minimal additional training would be an appropriate next step.

We have been examining motivational enhancement therapy (Miller \& Rollnick, 1993) for bulimia nervosa because we have been struck by the parallel course that clinical scientists in eating disorders and the addictions have been taking. By increasing patient motivation, it is hoped that compliance with treatment will also increase. As discussed earlier, in our study on self-treatment of bulimia nervosa, those who complied with treatment had a much better outcome than those who did not. Factors that increase compliance with treatment, therefore, would be expected to lead to a better treatment outcome.

Given the increasing prevalence of bulimia nervosa over the last 10-15 years, and the paucity of specialist centres, there is a particular need to make more effective interventions in primary care. Education about the typical presentation of eating disorders would be a start. For example, data from a nationwide general practitioner (GP) database showed that approximately one-third of patients with a GP diagnosis of bulimia nervosa had been prescribed laxatives, diuretics or other gastrointestinal medicines in the years before their diagnosis was recognised (Turnbull et al, submitted). Awareness that requests for such medications may be a marker of an eating disorder may lead to an earlier diagnosis. A booklet for GPs that gives details of diagnostic issues and early management is available (Myers et al, 1993) and a short training course for GPs has been developed to address this issue (Fairburn, personal communication).

\section{Other costs}

The cost of psychiatric treatment does not convey the full economic cost of bulimia nervosa (see Robinson, 1993b). Additional medical and dental expenses result directly from symptomatic behaviour (e.g. laxative abuse, vomiting) or indirectly from associated difficulties (e.g. self-harm, alcohol or drug misuse). In addition, there are indirect costs such as the effects on child care or loss of work. Families are often affected, with one or other parent taking time off work to act as carer. These costs are more or less difficult to evaluate, and different sources bear the costs of each. There are, however, methods available to address these difficulties (Robinson, 1993b), although this has not yet been done for eating disorders.

The advent of bulimia nervosa has significantly increased the cost of treating eating disorders and many questions remain. For example, are we witnessing a new demand on mental health care resources or is there a transfer of need from other areas of psychiatry? It may be that, in earlier years, women might have developed depression, while today, women develop bulimia nervosa. Even if a proportion of the increase in cost is due to a change in the form of psychological distress, it has fresh cost implications for the health service as the associated deterioration in physical health (of bulimia nervosa over, say, depression) places new demands on dental and medical services.

\section{Conclusion}

In conclusion, a great deal of progress has been made in developing effective interventions for bulimia nervosa. Comparison of abstinence rates with different treatments allows a cost-effectiveness analysis by purchasers quoted different prices. On present evidence, an approach using one of the selftreatment manuals that are now available, either as the first step of treatment (sequential) or as part of guided self-care, is a good beginning, both from a clinical and an economic standpoint. There is scope for further development of the sequential approach in primary care, as counsellors and GPs could implement and supervise the use of self-help manuals that detail specialist treatment strategies. A multi-centre trial would be necessary to explore this further, best planned in collaboration with a health economist. Drug treatment is probably an expensive option, given the relatively poor abstinence rates, but a comparative analysis of therapist versus GP costs would need to be included in any more definitive statement. Drug treatment may also have a role in supplementing psychotherapeutic treatment. However, other factors make further interpretation difficult. Normative data on the most effective treatment for each type of patient are not currently available, but are essential if a fully informed clinical and economic decision is to be made in individual cases. In the meantime, clinical judgement has to be our best guide. Our task is not to be daunted by the lack of accessible data, but to use the information available to implement effective treatment and to generate future studies that will include a planned economic evaluation alongside a clinical assessment. 


\section{Clinical implications}

- A sequential approach to treatment may be a useful strategy, both clinically and economically.

- There is a need for formal clinical trials, in which health costs are an integral part of the analysis.

\section{Limitations}

- The review compared studies that were not designed to include a cost component.

- We did not attempt a formal economic analysis.

- Our recommendations for future studies may themselves be costly and difficult to fund.

\section{References}

AGRAs, W. S. (1993) Short-term psychological treatments for binge eating. In Binge Eating: Nature, Assessment and Treatment (eds C. G. Fairburn \& G. T. Wilson), pp. 270-286. London: Guilford Press.

Cooper, P. J., Coker, S. \& Flemino, C. (1994) Self-help for bulimia nervosa: a preliminary report. International Journal of Eating Disorders, 16, 401-404.

FAIRBURR, C. G. (1995) Overcoming Binge Eating. New York: Guilford Press.

- Jones, R., Peveler, R. C., et al (1993) Poychotherapy and bulimia nervosa: longer-term effects of interpersonal poychotherapy, behaviour therapy and cognitive behaviour therapy. Archives of General Psychiatry, 50, 419-428.

-, Norman, P. A., Welch, S. L, et al (1995) A prospective study of outcome in bulimia nervoea and the long-term effects of three poychological treatments. Archives of General Psychiatry, 52, 304-312.

Fetres, P. A. \& Peters, J. M. (1992) A meta-analysis of group treatments for bulimia nervosa. International Journal of Eating Disorders, 11, 97-110.
Goldoten, D. J., Wrson, M. G., Thompson, V. L., et al (1995) Long term fluoxetine treatment of bulimia nervosa. British Journal of Psychiatry, 166, 660-666.

Howard, K. I., OrLinsky, D. E. \& Lueger, R. J. (1993) The design of clinically relevant outcome research: some considerations and an example. In Research Foundations for Psychotherapy Practice (eds M. Aveline \& D. Shapiro), pp. 215-231. Chichester: Wiley.

Jotnson, C., Tomen, D. \& Enright, A. B. (1989) Prevalence and clinical characteristics of borderline patients in an eating disordered population. Journal of Clinical Psychiatry, 50, 9-15.

LAessLe, R. G., ZoetTh, C. \& PTRKE, K. (1987) Meta-analysis of treatment studies for bulimia. International Journal of Eating Disorders, 6, 647-653.

MILER, W. R. \& RoLuNICK, S. (1993) Motivational Interviewing: Preparing People to Change Addictive Behaviours. New York: Guilford Press.

Myers, S., Davies, M. \& Treasure, J. (1993) a General Practitioner's Guide to Eating Disorders. London: Maudsley.

Ozmstend, M. P., DAvis, R., Rockert, W., et al (1991) Efficacy of a brief group paychooducational intervention for bulimia nervosa. Behavior Research and Therapy, 29, 71-83.

RATHNER, G., Borsch, C., MAURER, G., et al (1993) The impact of a 'Guided Self-Help Group' on bulimic women: a prospective 15 month study of attenders and non-attenders. Journal of Psychosomatic Research, 37, 389-396.

Rosmson, R. (1993a) Economic evaluation and health care. Costeffectiveness analysis. British Medical Journal, 307, 793-795.

(1993b) Economic evaluation and health care. Cost and cost-minimisation analysis. British Medical Journal, 307, 726728.

Treasure, J., SChmidt, U., Troop, N., et al (1996) A randomised controlled trial of sequential treatment for bulimia nervosa incorporating a self-care manual: outcome at end of treatment and at eighteen month follow-up. British Journal of Psychiatry, 168, 94-98.

Troop, N., SCHMIDT, U., TILLER, J., et al (1996) Compliance with a self-directed treatment manual for bulimia nervosa: predictors and outcome. British Journal of Clinical Psychology. In press.

TURnzull, S., Ward, A., Treasure, J. L., et al. Eating disorders in the primary care setting: any evidence of an increase? Submitted.

WEst, R. (1994) Eating Disorders: Anorexia Nervosa and Bulimia Nervosa. London: Office of Health Economics.

Janet L. Treasure, MRCPsych, Nicholas A. Troop, BSc, Anne Ward, MRCPsych, Eating Disorders Unit, Institute of Psychiatry, London

Correspondence: J. Treasure, Eating Disorders Unit, Institute of Psychiatry, De Crespigny Park, Denmark Hill, London SE5 8AF

(First received 18 December 1995, final revision 2 April 1996, accepted 19 April 1996) 